\title{
(-)-Epigallocatechin gallate attenuates NADPH-d/ nNOS expression in motor neurons of rats following peripheral nerve injury
}

\author{
I-Hua Wei ${ }^{1 *}$, Hui-Chin Tu ${ }^{4}$ Chih-Chia Huang ${ }^{2}$, Mang-Hung Tsai ${ }^{1}$, Chi-Yu Tseng ${ }^{3}$ and Jeng-Yung Shieh ${ }^{4 *}$
}

\begin{abstract}
Background: Oxidative stress and large amounts of nitric oxide (NO) have been implicated in the pathophysiology of neuronal injury and neurodegenerative disease. Recent studies have shown that (-)-epigallocatechin gallate (EGCG), one of the green tea polyphenols, has potent antioxidant effects against free radical-mediated lipid peroxidation in ischemia-induced neuronal damage. The purpose of this study was to examine whether EGCG would attenuate neuronal expression of NADPH-d/nNOS in the motor neurons of the lower brainstem following peripheral nerve crush. Thus, young adult rats were treated with EGCG $(10,25$, or $50 \mathrm{mg} / \mathrm{kg}$, i.p.) $30 \mathrm{~min}$ prior to crushing their hypoglossal and vagus nerves for 30 seconds (left side, at the cervical level). The treatment (precrush doses of EGCG) was continued from day 1 to day 6, and the animals were sacrificed on days 3, 7, 14 and 28. Nicotinamide adenine dinucleotide phosphate-diaphorase (NADPH-d) histochemistry and neuronal nitric oxide synthase (nNOS) immunohistochemistry were used to assess neuronal NADPH-d/nNOS expression in the hypoglossal nucleus and dorsal motor nucleus of the vagus.
\end{abstract}

Results: In rats treated with high dosages of EGCG (25 or $50 \mathrm{mg} / \mathrm{kg}$ ), NADPH-d/nNOS reactivity and cell death of the motor neurons were significantly decreased.

Conclusions: The present evidence indicated that EGCG can reduce NADPH-d/nNOS reactivity and thus may enhance motor neuron survival time following peripheral nerve injury.

\section{Background}

Peripheral nerve injury (PNI) produces numerous morphological, physiological, and biosynthetic changes in the damaged neurons [1-3]. Damage caused by PNI is associated with a variety of mechanisms, especially oxidative stress and subsequent massive production of oxygenderived free radicals, which are thought to play a major role in neurological disorders [4]. First identified as the endothelium-derived relaxing factor [5,6], nitric oxide is a short-lived free radical and gaseous biological messenger produced by nitric oxide synthase (NOS). This enzyme catalyzes the oxidation of $\mathrm{L}$-arginine and nicotinamide adenine dinucleotide phosphate (NADPH) by $\mathrm{O}_{2}$

\footnotetext{
* Correspondence: ihwei@mail.cmu.edu.tw; nadphd@ha.mc.ntu.edu.tw 'Department of Anatomy and Cell Biology, College of Medicine, China Medical University, Taichung, Taiwan

${ }^{4}$ Department of Anatomy and Cell Biology, College of Medicine, National Taiwan University, Taipei, Taiwan

Full list of author information is available at the end of the article
}

to yield L-citrulline and NO [7]. Three distinct NOS isoforms have been described: inducible (iNOS), endothelial (eNOS), and neuronal (nNOS) [8]. In the nervous system, free radical gas plays an important role in the regulation of physiological activities ranging from synaptic plasticity to neuroendocrine functions [9]. NO combines rapidly with superoxide to form peroxynitrite, a potent oxidizing agent with cytotoxic actions [10-12]. Previous studies have indicated that PNI in adult animals results in dramatic up-regulation of nitric oxide synthase in certain types of central and peripheral neurons that normally lack or hardly ever express the enzyme [13-18]. The injury-induced expression of NOS seems to signal the impending death of lesioned cells, as the enzyme acts as a killer protein by producing neurotoxic levels of NO [19-28]. Accordingly, numerous studies have examined, in various models of injury, the possibility of oxygen free radical scavengers as therapeutic agents for oxidative neuronal damage $[22,26,29]$.

\section{Biomed Central}


Green tea (Camellia sinensis) is one of the most popular beverages in the world because of its attractive flavor and aroma. It contains an abundance of so-called 'green tea polyphenols (GTPs), typically flavonoids or catechins, which are polyphenolic complexes with potential free radical scavenger activity. Many studies have shown that GTP can scavenge reactive oxygen species (ROS) such as hydroxyl radical $(\cdot \mathrm{OH})$, hydrogen peroxide $\left(\mathrm{H}_{2} \mathrm{O}_{2}\right)$, and superoxide anion $\left(\mathrm{O}_{2}{ }^{-}\right)$as well as reactive nitrogen species (RNS) such as nitric oxide $(\cdot \mathrm{NO})$ and peroxynitrite $\left(\mathrm{ONOO}^{-}\right)$in many systems. GTP also acts to protect cells from damage induced by the free radicals in the gaseous phase [30-35]. Green tea contains many polyphenolic compounds, including (-)-epigallocatechin gallate (EGCG), (-)-epigallocatechin (EGC), (-)-epicatechin gallate (ECG), and (-)-epicatechin (EC). Of these, EGCG is the most abundant and with two triphenolic groups, it is the most effective antioxidant [36,37]. Although the mechanisms of EGCG's antioxidant activity remain unclear, pharmacological studies have identified several antioxidant properties such as: (1) blockade of nNOS and iNOS induction $[34,38,39]$ and (2) scavenging of free radicals or attenuation of lipid peroxidation $[36,37,40]$. Thus, EGCG is expected to be neuroprotective.

To our knowledge, the potential effects of EGCG on NOS activity and neuronal damage following nerve injury in vivo remain to be explored. Notably, most previous studies concerning the effects of EGCG on NOS activity are biochemical rather than morphological. Thus, we carried out a morphological study using nicotinamine adenine dinucleotide phosphate-diaphorase (NADPH-d) histochemistry along with nNOS immunohistochemistry to explore the time-course of nNOS expression in the hypoglossal nucleus (HN) and dorsal motor nucleus (DMN) of the vagus in rats subjected to peripheral nerve crush injury (PNCI) and to elucidate the effect of post-PNCI EGCG treatment on motor neurons in the lower brainstem.

\section{Methods}

\section{Treatment of experimental animals}

Adult male Wistar rats $(\mathrm{n}=80)$ weighing $200-250 \mathrm{~g}$ were used and obtained from the Laboratory Animal Center of the National Taiwan University. We followed the Guide for the Care and Use of Laboratory Animals (1985) as stated in the United States NIH guidelines (NIH publication no.86-23) to treat animals for this study including the care, housing, handling and experimental procedures. All the experiments were approved by our Laboratory Animal Center, China Medical University, Taiwan. All efforts were made to minimize both the animal suffering and the number of animals used for this investigation.

The experimental animals were anesthetized with an intramuscular (i.m.) injection of mixtures of zoletil
(30 mg/kg) and xylazine (10 mg/kg). After anesthesia, the left vagus and the hypoglossal nerves were subjected to crush injury by clamping with a small hemostatic forceps for $30 \mathrm{~s}$; for the former, the level of clamping was mid-cervical, whereas for the latter, right below the tendon of the digastric muscle. In sham-operated animals (controls), similar procedures were carried out except both of the left side nerves remained intact. The experimental animals were divided into four groups: I, II, III, and IV (10, 25, $50 \mathrm{mg} / \mathrm{kg}$ EGCG-pretreatment + PNCI and PNCI only, respectively). Previous in vivo studies have demonstrated that EGCG can pass the blood-brain barrier and reach the brain parenchyma $[34,38,41,42]$. Animals in each group were therefore received daily intraperitoneal injections of EGCG or normal saline for successive six days with the last injection at $30 \mathrm{~min}$ before PNCI. Different doses (10, 25 , or $50 \mathrm{mg} / \mathrm{kg}$ body weight dissolved in normal saline) of EGCG (cat No.E4143, Sigma-Aldrich, St Louis, MO, USA) were applied in the present study. Each experimental group was subdivided into four groups $(n=5)$ and sacrificed at 3, 7, 14, and 28 days. All the experimental animals were housed under the same conditions (controlled temperature $\left[22^{\circ} \mathrm{C}\right]$ and 12 -h light/dark cycle) and given free access to food and water ad libitum.

\section{Perfusion and tissue preparation}

At each time point, rats were deeply anesthetized with mixtures of zoletil $(30 \mathrm{mg} / \mathrm{kg})$ and xylazine $(10 \mathrm{mg} / \mathrm{kg})$ and perfused transcardially with $100 \mathrm{ml}$ of Ringer's solution followed by $300 \mathrm{ml}$ of $4 \%$ paraformaldehyde in 0.1 $\mathrm{M}$ phosphate buffer (PB), $\mathrm{pH}$ 7.4. After perfusion, the brainstem and its nuclei were quickly removed, rinsed in $0.1 \mathrm{M} \mathrm{PB}$, and transferred through a series of increasing concentrations of sucrose buffer (10 - 30\%) for cryoprotection at $4^{\circ} \mathrm{C}$ overnight. Serial $30-\mu \mathrm{m}$ thick sections of the brainstem were cut transversely with a cryostat on the following day and were alternately placed at a distance of $120 \mu \mathrm{m}$ apart into four-well dishes. Sections collected in the first and second wells were processed for NADPH-d histochemistry and counterstained with neutral red; sections in the third well were processed for nNOS immunohistochemistry and those in the fourth well were processed for nNOS immunofluorescence labeling combined with NADPH-d histochemistry.

\section{NADPH-d histochemistry}

NADPH-d used as a selective marker for nNOS as described previously $[21,27,43,44]$. Briefly, sections in the first two wells were incubated with NADPH-d medium $(0.1 \mathrm{mg} / \mathrm{ml}$ nitroblue tetrazolium, $1 \mathrm{mg} / \mathrm{ml}$ $\beta$-NADPH, and $0.3 \%$ Triton $\mathrm{X}-100$ in $0.1 \mathrm{M} \mathrm{PB}[\mathrm{pH}$ 7.4]) for $1 \mathrm{~h}$ at $37^{\circ} \mathrm{C}$, washed several times in $0.1 \mathrm{M} \mathrm{PB}$ to terminate the reaction. For counting neuronal numbers, sections from the second well were further 
counterstained with neutral red, dehydrated through a graded series of alcohols, cleared with xylene, and coverslipped with Permount.

\section{nNOS immunohistochemistry}

Sections in the third well were rinsed in $0.01 \mathrm{M}$ phosphate buffered saline (PBS), $\mathrm{pH} 7.4$, treated with $0.01 \mathrm{M}$ PBS containing $10 \%$ methanol and $3 \%$ hydrogen peroxide for $1 \mathrm{~h}$ to abolish the endogenous peroxidase activity, rinsed 3 times with PBS, and incubated with medium containing $3 \%$ normal horse serum, $2 \%$ bovine serum albumin, and $0.1 \%$ Triton $\mathrm{X}-100$ for $1 \mathrm{~h}$. The reacted sections were then washed several times with PBS, incubated with antibody to nNOS (1:100; Santa Cruz Biotechnology, Burlingame, CA, USA) for $24 \mathrm{~h}$ at $4^{\circ} \mathrm{C}$, treated with biotinylated secondary antibody (1:200; Vector Laboratories, Burlingame, CA, USA) for $1 \mathrm{~h}$ at room temperature, and incubated with Streptavidin/HRP (DAKO A/S, Glostrup, Denmark). The signal was developed with diaminobenzidine (a peroxidase substrate).

\section{Colocalization of nNOS and NADPH-d reactivity}

Colocalization of NADPH-d and nNOS was detected by immunofluorescence labeling of nNOS followed by histochemical staining for NADPH-d $[21,27,43,44]$. Sections in 4-well dishes were rinsed in 0.01 M PBS, incubated in medium containing 3\% normal goat serum, $2 \%$ bovine serum albumin, and $0.1 \%$ Triton $\mathrm{X}-100$ for $1 \mathrm{~h}$ to block nonspecific binding, rinsed with PBS, and incubated with mouse monoclonal antibody against nNOS (1:100; Santa Cruz Biotechnology) for $24 \mathrm{~h}$ at $4{ }^{\circ} \mathrm{C}$. The reacted sections were then treated with fluorescein isothiocyanate (FITC)conjugated anti-rabbit secondary antibody (1:200; Vector Laboratories) for $2 \mathrm{~h}$ at room temperature, washed several times with PBS, and coverslipped with buffered glycerin to prevent fading. The tissue slides were photographed using a ZEISS fluorescence microscope equipped with an appropriate excitation filter (450-490 nm) for observing FITC-labeled nNOS-immunoreactive neurons. After photographing, the sections were again washed several times again in $0.1 \mathrm{M} \mathrm{PB}$, processed for NADPH-d histochemistry (as described above), rapidly dehydrated through a graded series of alcohols, cleared with xylene, and coverslipped with Permount.

\section{Quantitative study and image analysis}

In each animal, 20-30 sections representing the entire length of the $\mathrm{HN}$ and DMN (extending from the obex $1.8 \mathrm{~mm}$ caudally and $1 \mathrm{~mm}$ rostrally) were collected for NADPH-d histochemistry and neutral red counterstaining and cell counting. To avoid bias for the counts, large neurons $(25-50 \mu \mathrm{m})$ with clearly outlined nuclei but not small motor neurons were counted due to the latter could not be distinguished from interneurons being 10-18 $\mu \mathrm{m}$ in diameter. Both the NADPH-d positive and negative (neutral red-counterstained) cells in the $\mathrm{HN}$ and DMN, either ipsilateral or contralateral to the nerve crush site, were counted and summed to present the total number of cells in 20-30 sections (unit volume, $u$ ). The labeling percentage was calculated by dividing the number of NADPH-d(+) neurons on the lesion side by the total number of neurons on the same side. To confirm the coexistence of $\mathrm{NADPH}-\mathrm{d}(+)$ and $\mathrm{nNOS}(+)$ neurons, double-labeled neurons in the $\mathrm{HN}$ and DMN of the same sections were counted on the photomicrographs (data not shown). Cells containing nNOS were counted in the sections processed for NADPH-d histochemistry since neuronal NADPH-d is a reliable marker of nNOS $[21,43]$. Besides, the results of neuronal staining in sections stained for NADPH-d are usually better than nNOS, which in turn facilitates computer-assisted quantitative assessment. Labeling intensity of NADPH$\mathrm{d}(+)$ neurons was quantified using a computer-based image analysis system (MGDS) and Image Pro-Plus software (Media Cybernetics, Silver Spring, MD, USA). Labeled sections were scanned at $100 \times$ magnification in bright field using a digital camera mounted on a ZEISS microscope and the images were displayed on a high-resolution monitor. Up to 100 cells per section were measured along the entire length of the $\mathrm{HN}$ and DMN. At $100 \times$ magnification, the cytoplasmic optical density (OD), which was used as an index of labeling intensity, in NADPH-d(+) neurons was measured by tracing the contour of the labeled soma in digitized images. The background OD in each section was measured by averaging five random polygons (area of polygon $=150 \mu \mathrm{m}^{2}$ ) within the neuropil of the corresponding $\mathrm{HN}$ and $\mathrm{DMN}$ regions on the non-lesioned side. The staining intensity in a tissue section reflected the amount of enzyme activity. Thus, all parameters were controlled using methods to ensure consistent gray level adjustment, histogram stretch, and minimal optical density. To avoid introducing bias, two observers blinded to the animal treatment group counted cells in the NADPH-d stained sections and evaluated the data from image analysis of $\mathrm{HN}$ and $\mathrm{DMN}$ neurons.

\section{Statistical analysis}

All data of this study were expressed as mean \pm SEM and statistical significance was determined with a commercially available software package (SPSS version 12; SPSS, Chicago, IL). Between-group differences in the percentage and OD of NADPH-d positive neurons at various time points, with or without EGCG treatment, were evaluated using one-way analysis of variance (ANOVA) followed by LSD post hoc test. The data collected in experimental 
groups within the same time point (i.e., $10 \mathrm{mg} / \mathrm{kg}$ EGCG pretreated versus control non-treated group) were further analyzed using Student's $t$ test. Statistical difference was considered significant if $p<0.05$.

\section{Control experiments}

Some negative controls were included to ensure the validity of the NADPH-d histochemical and nNOS immunohistochemical results. Thus, the medium without $\beta$-NADPH was used as the control in NADPH-d staining protocol, whereas media without primary or secondary antibodies were used as controls in nNOS immunochemical procedure.

\section{Results}

\section{NADPH-d/nNOS colocalization studies}

Labeling for both nNOS and NADPH-d showed that the majority of the NADPH-d positive neurons in the HN and DMN were also positive for nNOS (Figure 1). The distribution of nNOS-positive neurons in the brainstem motor nuclei paralleled that of NADPH-d positive neurons. The time course of PNCI-induced nNOS expression in the HN and DMN on the lesion side coincided with that of PNCI-induced NADPH-d expression. Since the NADPH-d histochemical method was sensitive and specific, it was used for both qualitative and quantitative analyses. No NADPH-d and nNOS positive neurons were detected in the $\mathrm{HN}$ or the DMN of negative control rats.
NADPH-d/nNOS expression in the hypoglossal nucleus of EGCG-treated rats following $\mathrm{PNCl}$

In sham-operated groups of both non-treated and EGCGtreated rats, no NADPH-d/nNOS positive [NADPH-d/ $\mathrm{nNOS}(+)$ ] neurons were found on either side of the hypoglossal nucleus $(\mathrm{HN})$. In non-treated rats subjected to PNCI, NADPH-d/nNOS(+) neurons were first detected in the $\mathrm{HN}$ at 3 days, with their number reaching a peak at 7 days ( $p<0.05$ vs. various time points; Figures $2 \mathrm{~A}, 3 \mathrm{~A}$ and 4; Table 1$)$. The percentage of NADPH-d/nNOS(+) neurons increased steeply from $4.92 \pm 0.29 \%$ at 3 days to $28.64 \pm 0.93 \%$ at 7 days after PNCI (Figure 4 ) but then declined to $19.45 \pm 0.95 \%$ and $3.69 \pm 0.28 \%$ at 14 and 28 days, respectively, in rats with longer survival times (Figures 2D, 3D and 4). It is also true for the changes of cell total number that showed an increased population of cells at various time points and reached the climax at 7 days $(370 \pm 9$ cell $/ \mathrm{u}$; Table 1$)$ after PNCI. The distribution of $\mathrm{NADPH}-\mathrm{d} / \mathrm{nNOS}(+)$ neurons was random (Figures 2 and 3 ) and, at 3 days after PNCI, the staining intensity of most labeled neurons was weak, but gradually enhanced to $292 \pm 35 \%$ above background values at 7 days following PNCI (Figure 5). At the same time, shrunken NADPH-d intensely-labeled neurons occurred (Figures 2 and 3).

In rats receiving high dosages of EGCG (25 and $50 \mathrm{mg} / \mathrm{kg}$ ) treatment then subjected to PNCI, their staining pattern of NADPH-d/nNOS was paralleled to those of non-treated ones, but their total number and
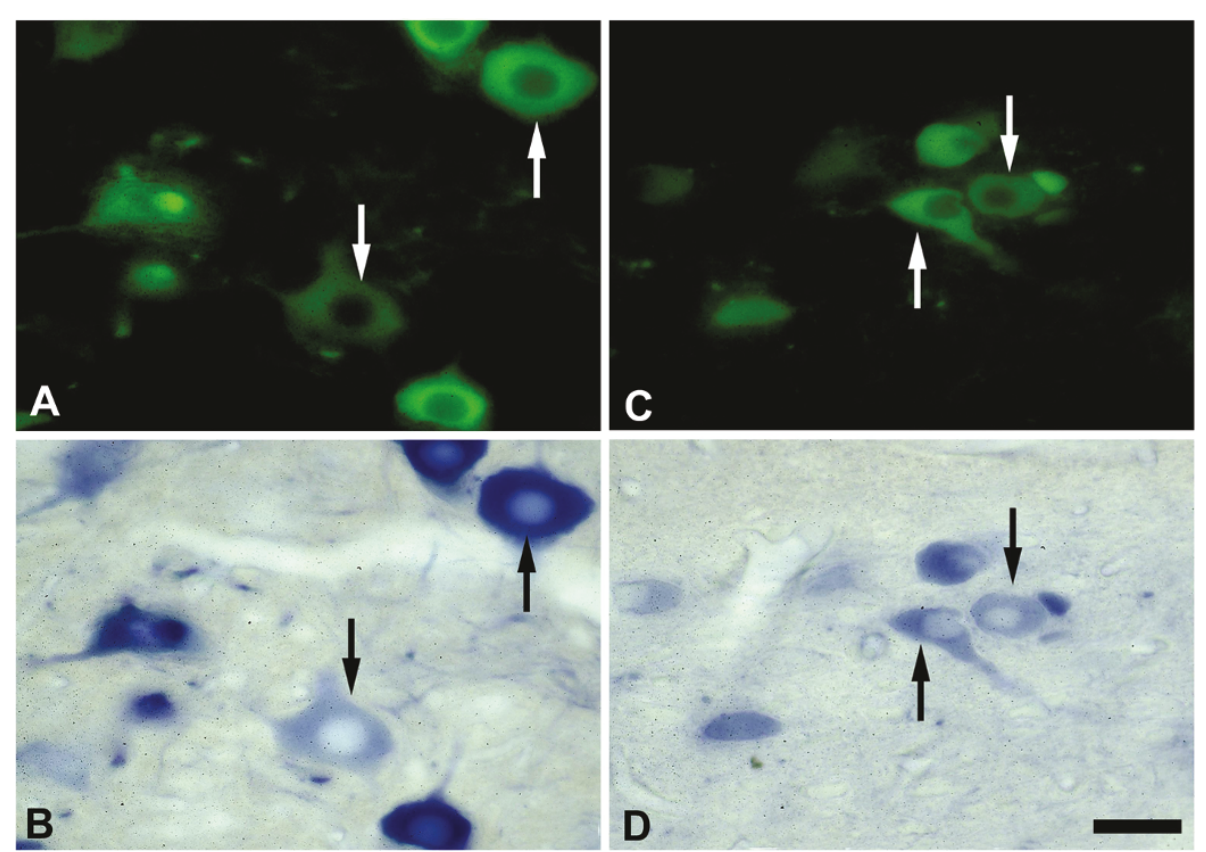

Figure 1 NADPH-d/nNOS colocalization studies. Fluorescence $(A, C)$ and light (B, D) photomicrographs showing nNOS(+) (A, C) and NADPH-d $(+)(B, D)$ labeled neurons on the lesioned side of the HN (A, B) and DMN (C, D) of rats following crush injury of left vagus and hypoglossal nerves. Note that a most of nNOS(+) neurons (arrows in A-D) contain NADPH-d in the HN and DMN. Scale bar $=50 \mu \mathrm{m}$. 


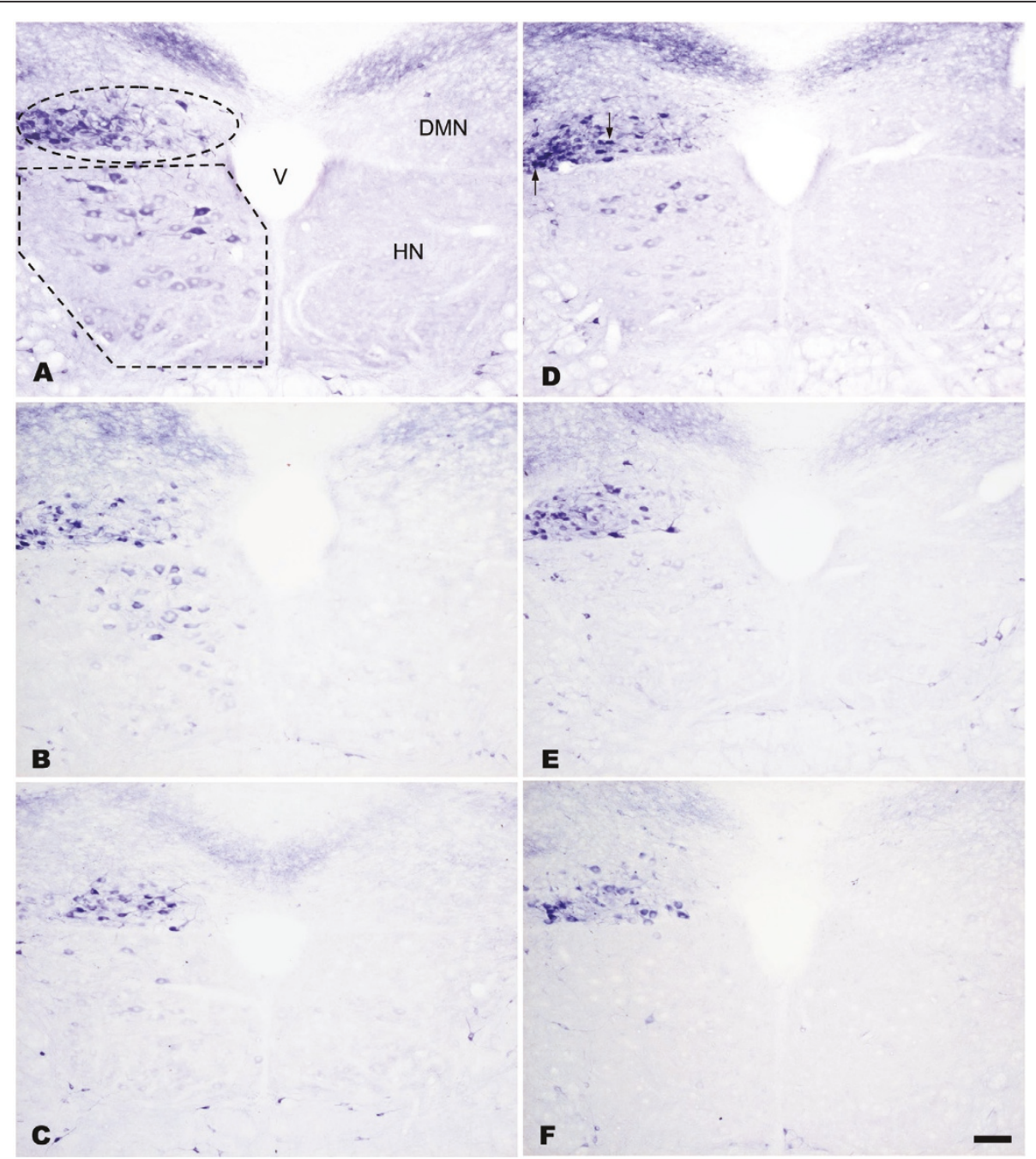

Figure 2 Histochemistry of NADPH-d in the HN and DMN. Light photomicrographs showing the injury-induced NADPH-d reactivity in the lesioned side of the HN and DMN at $7(A, B, C)$ and $14(D, E, F)$ days after PNCI. NADPH-d staining is barely detected in HN and DMN neurons in the contralateral intact side of the nuclei (the right side of each figure). Note the marked increase in NADPH-d reactivity both in terms of staining intensity and number of labeled neurons at 7 days in the $\mathrm{HN}$ and DMN after PNCI (A, B, C). Also note that the population of NADPH-d $(+)$ neurons in the $\mathrm{HN}$ is reduced at 14 days after $\mathrm{PNCl}$, whereas that in the DMN tends to increase $(\mathrm{D}, \mathrm{E}, \mathrm{F})$ when compared those at 7 days post PNCl. Some shrunken cells ( $\mathrm{D}$, arrows) are found to display intense NADPH-d reactivity. In rats receiving EGCG treatment at high dose (50 $\mathrm{mg} / \mathrm{kg}, \mathrm{C}, \mathrm{F}$ ), the diaphorase reactivity and the number of positive neurons are noticeably reduced in relation to those of non-treated rats (A, D) or animals treated with lower dose EGCG $(10 \mathrm{mg} / \mathrm{kg}, \mathrm{B}, \mathrm{E})$. The ellipse and polygonal dotted line in A outlines the area of the HN and DMN, respectively. $\vee$, fourth ventricle; Scale bar $=100 \mu \mathrm{m}$.

percentage of the labeled neurons were comparatively lower than those of non-treated groups $(p<0.05$, Figures 2, 3 and 4, Table 1). Similarly, EGCG-treated (25 and $50 \mathrm{mg} / \mathrm{kg}$ ) rats at various time points after PNCI showed a marked increase in the total number of NADPH-d negative cells $(p<0.05$ vs. non-treated groups; Table 1). The percentage of NADPH-d/nNOS $(+)$ neurons was slightly increased from $3.82 \pm 0.27 \%$ and $2.26 \pm 0.65 \%$ at 3 days to $17.52 \pm 0.70 \%$ and $8.33 \pm$ $0.76 \%$ at 7 days respectively in EGCG-treated (25 and $50 \mathrm{mg} / \mathrm{kg}$ ) rats after PNCI (Figure 4). In EGCG-treated rats killed at longer survival periods after $\mathrm{PNCI}$, the percentage of NADPH-d/nNOS(+) neurons was gradually decreased at 14 days and approached to zero at 28 days (Figure 4). EGCG treatment did not alter NADPH-d/ nNOS(+) neuron distribution but statistical evaluation revealed that the staining intensity of NADPH-d/nNOS appeared to be lower in the treated groups than nontreated ones at all time intervals post-injury $(p<0.05$, Figure 5). Low-dose (10 mg/kg) EGCG treatment had no effect on enzyme amount and expression (Figures 2B, D, 3B, D, 4 and 5; Table 1). 


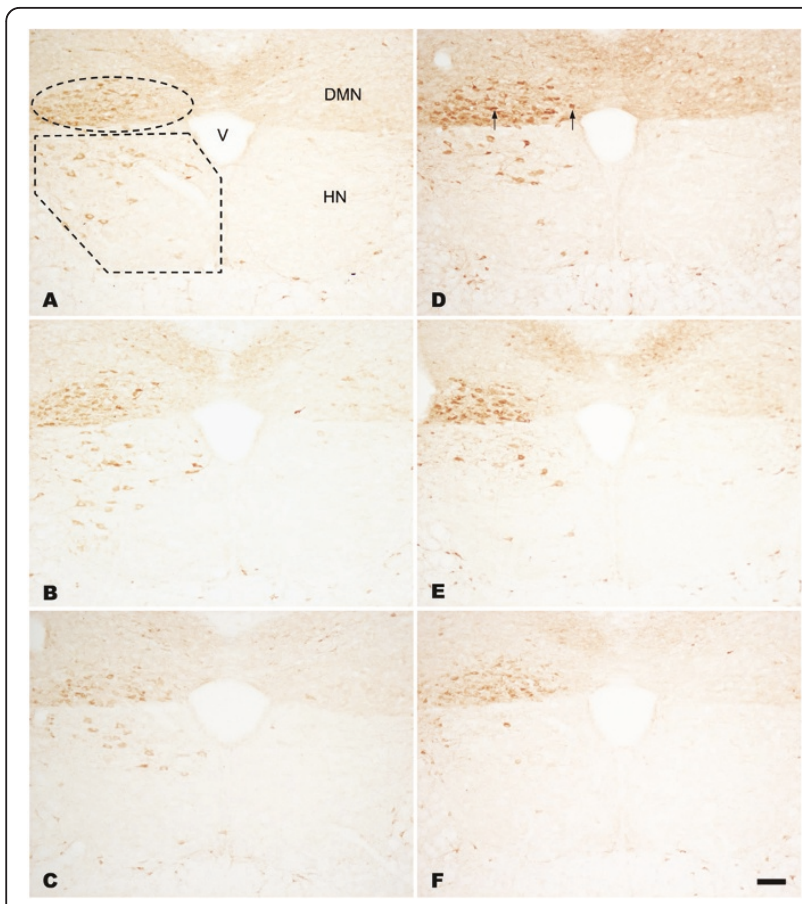

Figure 3 Immunohistochemistry of $\mathrm{nNOS}$ in the HN and DMN. Light photomicrographs showing the nNOS reactivity in the lesioned side of the $H N$ and $D M N$ at $7(A, B, C)$ and $14(D, E, F)$ days after $\mathrm{PNCl}$. nNOS staining is barely detected in $\mathrm{HN}$ and DMN neurons in the contralateral intact side of the nuclei (the right side of each figure), but significantly increased at 7 days in the affected HN and DMN after PNCl (A, B, C). When compared with those at 7 days post $\mathrm{PNCl}$, nNOS positive neurons in the $\mathrm{HN}$ are obviously decreased in number at 14 days following $\mathrm{PNCl}$, whereas those in the DMN are inclined to increase $(D, E, F)$. The immunoreactivities of nNOS are markedly declined in the lesioned $\mathrm{HN}$ and DMN of rats treated with high dose EGCG $(50 \mathrm{mg} / \mathrm{kg}, \mathrm{C}, \mathrm{F})$ in relation to those of non-treated rats $(A, D)$ or animals treated with lower dose EGCG (10 mg/kg, B, E). Similarly, there were some nNOS intensely-labeled neurons appear shrunken in contour ( $\mathrm{D}$, arrows) following PNCI. $\mathrm{V}$, fourth ventricle; Scale bar $=100 \mu \mathrm{m}$.

\section{NADPH-d/nNOS expression in the dorsal motor nucleus of} vagus of EGCG treated rats following $\mathrm{PNCl}$

In sham-operated groups of the rats treated with or without EGCG, hardly any detected NADPH-d/nNOS (+) neurons were found either on the left or right side of the DMN. From 3 days after PNCI, NADPH-d/nNOS (+) neurons were detected and randomly distributed throughout the injured DMN (Figures 2 and 3). There were some shrunken cells expressing intense NADPH-d at 14 days following PNCI (Figures 2 and 3). At the same time, treatment with high doses of EGCG (25 and $50 \mathrm{mg} / \mathrm{kg}$ ) tended to decrease the total number and percentage of NADPH-d/nNOS(+) neurons in the DMN $(462 \pm 13 \mathrm{cell} / \mathrm{u} ; 37.62 \pm 1.09 \%$ and $433 \pm 18 \mathrm{cell} / \mathrm{u}$; $35.06 \pm 1.31 \%$, respectively) when compared with the non-treatment one $(628 \pm 21 \mathrm{cell} / \mathrm{u} ; 53.08 \pm 0.91 \%$; Figure 4 and Table 1). The total number of NADPH-d
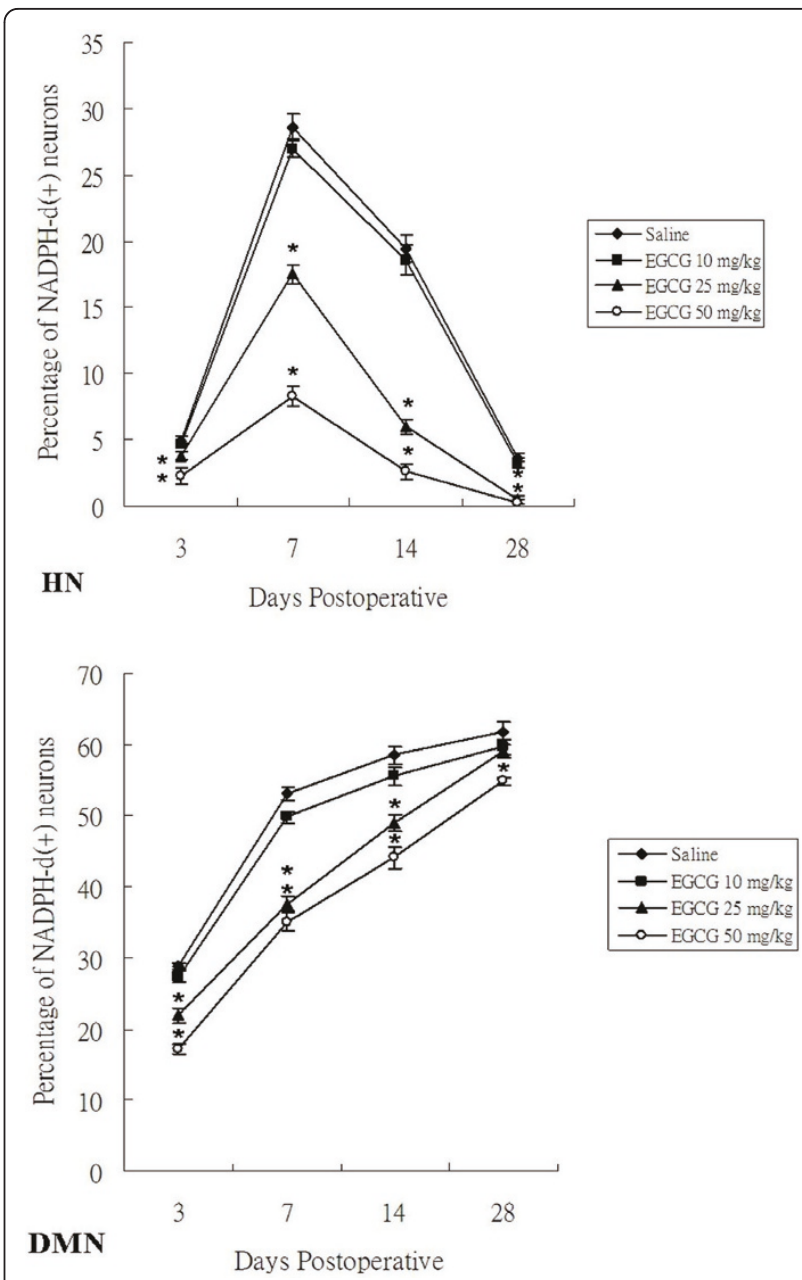

Figure 4 Alterations of NADPH-d reactivity in the HN and DMN at 3, 7, 14 and 28 days after PNCl and EGCG treatment.

Quantitative analysis shows that PNCl causes a remarkably-increased population of $\mathrm{NADPH}-\mathrm{d}(+)$ neurons at different time intervals examined. PNCl-induced NADPH-d(+) neurons reach their population peak at 7 days in the HN but continue to increase their population in the DMN until the end of the examination. Note a significant decrease of $\mathrm{NADPH}-\mathrm{d}(+)$ neurons in the groups receiving EGCG treated animals when compared with those of non-treated (salinetreated) ones at 7 and 14 days in the HN. At the same time intervals in the DMN, the groups receiving high dosages of EGCG (25 and 50 $\mathrm{mg} / \mathrm{kg}$ ) show a marked reduction of $\mathrm{NADPH}-\mathrm{d}(+)$ neuron population when compared with those receiving lower dose of EGCG (10 mg/ $\mathrm{kg}$ ) or saline. ${ }^{*}, p<0.05$ for the comparison between EGCG-treated and saline-treated groups at the same survival time point.

negative cells also showed a significantly increased in EGCG-treated (25 and $50 \mathrm{mg} / \mathrm{kg}$ ) rats after PNCI at various time points ( $p<0.05$ vs. non-treated groups; Table 1). Quantitative analysis of labeling intensity showed stronger diaphorase reactivity in cells of nontreated rats than those in rats treated with high-dose EGCG (25 and $50 \mathrm{mg} / \mathrm{kg}$ ) at 3, 7, and 14 days after PNCI $(p<0.05$, Figure 5$)$. However, at 28 days after $\mathrm{PNCI}$, the percentage of NADPH-d $(+)$ neurons on the 
Table 1 Effect of EGCG treatment on the total number of NADPH-d positive $(+)$ and negative $(-)$ cells in the lesioned side of DMN and $\mathrm{HN}$ at 3, 7, 14 and 28 days following $\mathrm{PNCl}$

\begin{tabular}{|c|c|c|c|c|c|c|c|c|}
\hline \multirow[t]{3}{*}{ Days postoperative } & \multicolumn{8}{|c|}{ Hypoglossal nucleus } \\
\hline & \multicolumn{2}{|c|}{ Saline } & \multicolumn{2}{|c|}{ EGCG (10 mg/kg) } & \multicolumn{2}{|c|}{ EGCG $(25 \mathrm{mg} / \mathrm{kg})$} & \multicolumn{2}{|c|}{ EGCG $(50 \mathrm{mg} / \mathrm{kg})$} \\
\hline & + & - & + & - & + & - & + & - \\
\hline 3 & $69 \pm 4$ & $1336 \pm 7$ & $65 \pm 3$ & $1343 \pm 7$ & $53 \pm 4$ & $1344 \pm 9$ & $31 \pm 4^{*}$ & $1368 \pm 7^{*}$ \\
\hline 7 & $370 \pm 9$ & $924 \pm 22$ & $352 \pm 11$ & $952 \pm 11$ & $238 \pm 10^{*}$ & $1121 \pm 11^{*}$ & $114 \pm 10^{*}$ & $1251 \pm 16^{*}$ \\
\hline 14 & $235 \pm 9$ & $963 \pm 34$ & $229 \pm 11$ & $1009 \pm 29$ & $79 \pm 8^{*}$ & $1239 \pm 10^{*}$ & $35 \pm 7^{*}$ & $1296 \pm 12^{*}$ \\
\hline 28 & $43 \pm 3$ & $1122 \pm 19$ & $38 \pm 4$ & $1137 \pm 31$ & $6 \pm 4^{*}$ & $1284 \pm 6^{*}$ & $4 \pm 3^{*}$ & $1297 \pm 5^{*}$ \\
\hline \multirow[t]{3}{*}{ Days postoperative } & \multicolumn{8}{|c|}{ Dorsal motor nucleus of vagus nerve } \\
\hline & \multicolumn{2}{|c|}{ Saline } & \multicolumn{2}{|c|}{ EGCG $(10 \mathrm{mg} / \mathrm{kg})$} & \multicolumn{2}{|c|}{ EGCG $(25 \mathrm{mg} / \mathrm{kg})$} & \multicolumn{2}{|c|}{ EGCG $(50 \mathrm{mg} / \mathrm{kg})$} \\
\hline & + & - & + & - & + & - & + & - \\
\hline 3 & $368 \pm 9$ & $909 \pm 6$ & $351 \pm 11$ & $934 \pm 8$ & $283 \pm 12^{*}$ & $1009 \pm 18^{*}$ & $222 \pm 9^{*}$ & $1067 \pm 10^{*}$ \\
\hline 7 & $628 \pm 21$ & $554 \pm 3$ & $590 \pm 14$ & $595 \pm 9$ & $462 \pm 13^{*}$ & $765 \pm 17^{*}$ & $433 \pm 18^{*}$ & $802 \pm 13^{*}$ \\
\hline 14 & $616 \pm 12$ & $439 \pm 20$ & $599 \pm 13$ & $480 \pm 17$ & $580 \pm 11$ & $603 \pm 19^{*}$ & $525 \pm 17^{*}$ & $667 \pm 18^{*}$ \\
\hline 28 & $588 \pm 16$ & $363 \pm 15$ & $586 \pm 11$ & $397 \pm 14$ & $603 \pm 14$ & $418 \pm 12^{*}$ & $608 \pm 24$ & $482 \pm 17^{*}$ \\
\hline
\end{tabular}

*, $p<0.05$ when compared with the saline-treated group.

crush injured side pretreated with $25 \mathrm{mg} / \mathrm{kg}$ EGCG was similar to those with $10 \mathrm{mg} / \mathrm{kg}$ EGCG or none $(p<0.05$, Figure 4). Moreover, there was no significant decline in the total number, percentage or labeling intensity of $\mathrm{NADPH}-\mathrm{d} / \mathrm{nNOS}(+)$ neurons in rats given a low-dose $(10 \mathrm{mg} / \mathrm{kg}$ ) EGCG treatment (Figures 2B, E, 3B, E, 4 and 5; Table 1).

\section{Cell population change of in the HN and DMN after EGCG pretreatment and $\mathrm{PNCl}$}

In sham-operated rats irrespective of treatment, the number of motor neurons in the HN and DMN (about 1400 and 1300 cell/u, respectively) at all time points was not significantly different from that in the corresponding nuclei of the opposite side. In non-treated rats at 28 days following PNCI, about $18 \%$ and $26 \%$ neuronal loss were respectively observed in the $\mathrm{HN}$ and DMN (Table 2). However, in high-dose EGCG (25 and $50 \mathrm{mg} / \mathrm{kg}$ )-treated rats following PNCI, a significant attenuation of neuronal loss $(p<0.05$, Table 2$)$ was found when compared with those of nerve-crushed rats treated with nothing or low-dose EGCG (10 mg/kg). No contralateral (right) cell death was found in the shamoperated groups as a consequence of unilateral (left) nerve crush.

\section{Discussion}

The present study has provided the first morphological evidence that EGCG treatment may reduce damage caused by nerve crush-induced oxidative injury in brainstem motor neurons having different functions in adult rats. The protective effect of EGCG on injured neurons was dose-dependent. Taken together, our results suggest that the protective effect of higher-dose EGCG may be responsible for suppressing PNCI-induced NADPH-d/
nNOS expression in the HN and DMN by its higher antioxidant ability.

\section{Role of NADPH-d/nNOS expression in motor neurons after PNCI}

The current study revealed induced expression of NADPH-d/nNOS in the lower brainstem motor neurons of adult rats following PNCI. At higher doses (25 and $50 \mathrm{mg} / \mathrm{kg}$ ), EGCG significantly reduced the total number, percentage and enzyme activity of cells expressing NADPH-d/nNOS; this beneficial effect was more pronounced with injury progress. Ultimately, this reduction in NADPH-d/nNOS reactivity was markedly correlated with the diminution of motor neuron loss at 7, 14, and 28 days after PNCI. It is well documented that initial NO level and the time of exposure to excess NO, generated by NOS via the overstimulation of NMDA receptors, may account for neuronal damage following PNI $[45,46]$. NO itself or peroxynitrite $\left(\mathrm{ONOO}^{-}\right)$, which is formed when $\mathrm{NO}$ couples with the superoxide anion, can trigger a series of biochemical reactions that culminate in damage to mitochondria, nucleic acids, proteins, lipids, or DNA $[9,47,48]$. The coincidence of overt lesion-induced NADPH-d/nNOS expression with the death of injured motor neurons has therefore been found $[14,49,50]$. On the other hand, inhibition of NOS production in mice following spinal root avulsion has been shown to effectively reduce neuronal death and enhance peripheral nerve regeneration $[14,15,51]$. Our previous and other studies on the motor neurons of adult rats have also shown that PNI along with hypoxia or PNCI significantly up-regulated NADPH-d/ nNOS expression [17]. This up-regulation of enzyme expression is due to the production of excess NO that has detrimental effects on these neurons [23,27]. Interestingly, preconditioning treatment with mild hypoxic may induce 

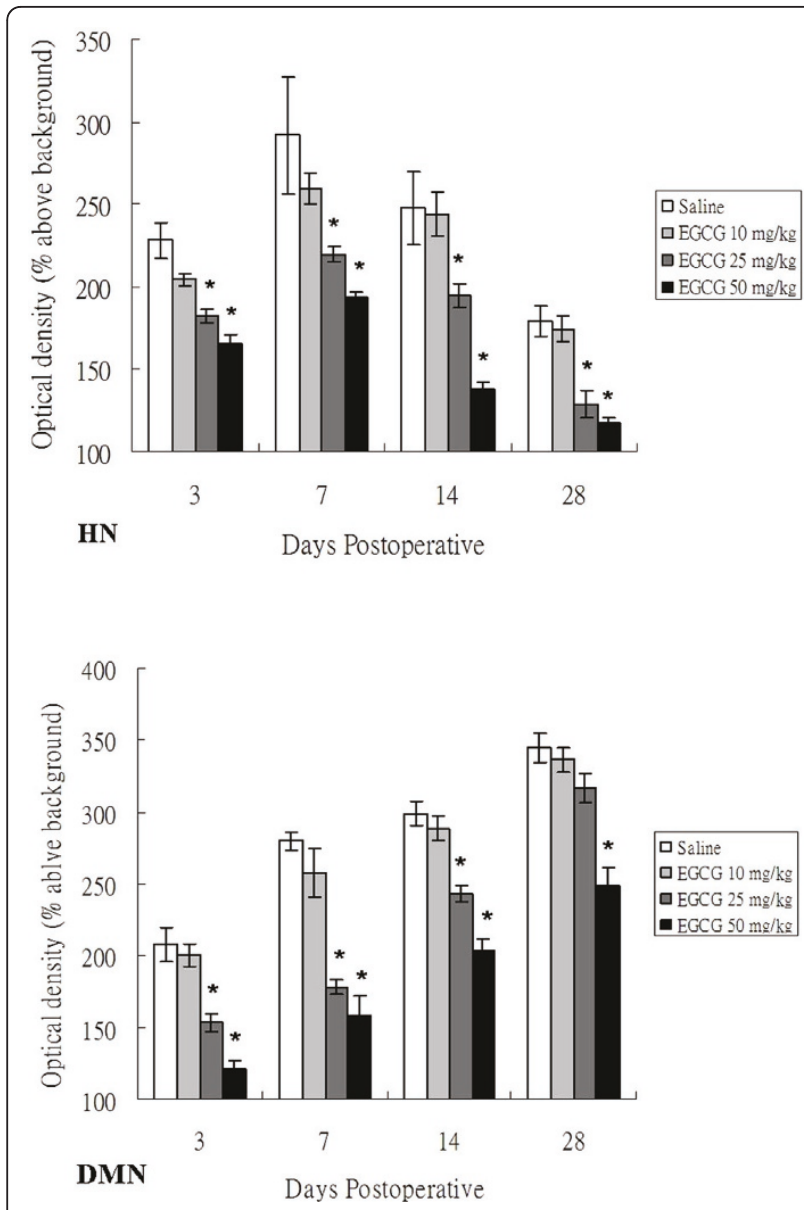

Figure 5 Mean optical density (OD) of NADPH-d(+) neurons in the $\mathrm{HN}$ and $\mathrm{DMN}$ of adult rats at 3, 7, 14 and 28 days after PNCI and EGCG treatment. The changes of mean optical density (determining staining intensity) in $\mathrm{NADPH}-\mathrm{d}(+)$ neurons of examined nuclei show a similar trend to the population modification of labeled neurons. The OD value in the $\mathrm{HN}$ of high dose EGCG-treated ( 25 and $50 \mathrm{mg} / \mathrm{kg}$ ) animals is much lower than those of low dose EGCG-treated $(10 \mathrm{mg} / \mathrm{kg})$ or non-treated ones at 7, 14 and 28 days after PNCI. The EGCG-mediated noticeable decrease of NADPH-d optical density is also evidenced in the DMN at different time points after $\mathrm{PNCl} .{ }^{*}, p<0.05$ for the comparison between EGCG-treated and saline-treated groups at the same survival time point.

small amount of NO that may alter the neuronal milieu and consequently protect against nerve crush-induced oxidative injury. Thus, whether NO is protective or destructive depends on its amount: the small amount would protect against neuronal damage and the large one cause cell death. In the present study, moderate and transient $\mathrm{NADPH}-\mathrm{d} / \mathrm{nNOS}$ expression was observed in the majority of $\mathrm{HN}$ neurons with $\mathrm{PNCI}$ progression during which mild neuronal death occurred. By contrast, NADPH-d/nNOS expression in DMN motor neurons after PNCI was prolonged and cell loss was pronounced with injury development (Figures 2, 3, and 7; Table 1 and 2). These results implied a close association of NADPH-d/nNOS expression with progressive neuronal death following PNCI. However, in lack of experimental evidence, the assumption that $\mathrm{NADPH}-\mathrm{d} / \mathrm{nNOS}$ positive neurons were the cells that died after the nerve injury may not necessarily be the case. The changes of NADPH-d or NOS activity may be part of a dynamic response to injury rather than simply pathologically related.

\section{Effects of EGCG on NADPH-d/nNOS expression in motor neurons following $\mathrm{PNCl}$}

Our previous and other studies have shown that green tea, melatonin, and hypoxic preconditioning strategies may enhance endogenous antioxidative defense systems against oxidative injury [20,22,23,26-28,52]. Green tea, rich in polyphenols, is one of the most widely consumed beverages in the world. EGCG is the most abundant catechin in tea and the major source of polyphenol bioactivity. Several studies have indicated that the antioxidant activity of EGCG may be neuroprotective and prevent NO-mediated neurological deficits. In in vitro studies, EGCG can scavenge cytotoxic NO or its downstream products [35,53]. The ability of EGCG to protect against neuronal damage and brain edema after unilateral cerebral ischemia and to reduce hippocampal neuronal damage after transient global ischemia in gerbils has been evidenced $[54,55]$. Our previous report also showed that EGCG may impart the suppressive effect on susceptibility of nodose neurons to nNOS-mediated neuropathy subsequent to severe hypoxic exposure [26]. It has been suggested that NO produced by nNOS and iNOS plays an important role in pathophysiologic processes including neurotoxicity and delayed neuronal death $[47,56]$. Consequently, the amount of NOS reactivity may be used as an index of neuronal damage severity. The present results clearly indicated that high-doses EGCG (25 and $50 \mathrm{mg} / \mathrm{kg}$ ) reduced NADPH-d/nNOS expression and neuronal loss following PNCI that caused excessive and prolonged expression of NADPH-d/nNOS leading to neurodestruction in the region studied. Reducing the activity of this enzyme (e.g., by treatment with EGCG) could decrease neurological signs and neuronal damage as reflected by evidences of our previous and other studies. All these results showed that nNOS inhibitors, melatonin, and high-doses EGCG (25 and $50 \mathrm{mg} / \mathrm{kg}$ ) reduced nNOS activity and neuronal damage after brain ischemia, hypobaric hypoxia, and PNI $[20,26,54,57]$. Similarly, the present study found that high-doses EGCG ( 25 and $50 \mathrm{mg} / \mathrm{kg}$ ) may be neuroprotective and thereby attenuate neuronal damage after peripheral nerve crush injury.

Possible mechanisms regarding the differential responses of different motor neurons to EGCG treatment after PNCI Our previous and other studies found differences in PNIinduced NADPH-d/nNOS expression between motor 
Table 2 Effect of EGCG treatment on the number of surviving motoneurons and percentage of cell loss in the lesioned side of DMN and $\mathrm{HN}$ at 3, 7, 14 and 28 days following $\mathrm{PNCl}$

\begin{tabular}{|c|c|c|c|c|c|}
\hline \multirow[t]{2}{*}{ Days postoperative } & \multicolumn{5}{|c|}{ Hypoglossal nucleus } \\
\hline & Sham & Saline & $\begin{array}{c}\text { EGCG } \\
(10 \mathrm{mg} / \mathrm{kg})\end{array}$ & $\begin{array}{c}\text { EGCG } \\
(25 \mathrm{mg} / \mathrm{kg})\end{array}$ & $\begin{array}{c}\text { EGCG } \\
(50 \mathrm{mg} / \mathrm{kg})\end{array}$ \\
\hline \multirow[t]{2}{*}{3} & $1416 \pm 11$ & $1405 \pm 8$ & $1408 \pm 9$ & $1398 \pm 8$ & $1399 \pm 9$ \\
\hline & - & $1 \pm 0.6 \%$ & $1 \pm 0.6 \%$ & $1 \pm 0.8 \%$ & $1 \pm 0.9 \%$ \\
\hline \multirow[t]{2}{*}{7} & $1408 \pm 10$ & $1294 \pm 18$ & $1304 \pm 17$ & $1360 \pm 11^{*}$ & $1365 \pm 7^{*}$ \\
\hline & - & $9 \pm 1.7 \%$ & $8 \pm 1.9 \%$ & $4 \pm 1.2 \%^{*}$ & $4 \pm 1.0 \% *$ \\
\hline \multirow[t]{2}{*}{14} & $1411 \pm 11$ & $1212 \pm 31$ & $1238 \pm 20$ & $1318 \pm 10^{*}$ & $1331 \pm 7^{*}$ \\
\hline & - & $14 \pm 2.0 \%$ & $12 \pm 2.0 \%$ & $7 \pm 1.0 \% *$ & $6 \pm 1.0 \% *$ \\
\hline \multirow[t]{2}{*}{28} & $1397 \pm 8$ & $1165 \pm 20$ & $1175 \pm 30$ & $1291 \pm 5^{*}$ & $1301 \pm 5^{*}$ \\
\hline & - & $18 \pm 2.0 \%$ & $17 \pm 2.0 \%$ & $9 \pm 1.0 \% *$ & $8 \pm 1.0 \%{ }^{*}$ \\
\hline \multirow[t]{2}{*}{ Days postoperative } & \multicolumn{5}{|c|}{ Dorsal motor nucleus of vagus nerve } \\
\hline & Sham & Saline & $\begin{array}{c}\text { EGCG } \\
(10 \mathrm{mg} / \mathrm{kg})\end{array}$ & $\begin{array}{c}\text { EGCG } \\
(25 \mathrm{mg} / \mathrm{kg}) \\
\end{array}$ & $\begin{array}{c}\text { EGCG } \\
(50 \mathrm{mg} / \mathrm{kg})\end{array}$ \\
\hline \multirow[t]{2}{*}{3} & $1284 \pm 10$ & $1277 \pm 13$ & $1286 \pm 8$ & $1293 \pm 8$ & $1289 \pm 7$ \\
\hline & - & $1 \pm 0.5 \%$ & $0 \pm 0.7 \%$ & $0 \pm 0.6 \%$ & $0 \pm 0.7 \%$ \\
\hline \multirow[t]{2}{*}{7} & $1292 \pm 8$ & $1182 \pm 19$ & $1185 \pm 16$ & $1227 \pm 11^{*}$ & $1235 \pm 8^{*}$ \\
\hline & - & $8 \pm 1.5 \%$ & $8 \pm 1.6 \%$ & $4 \pm 0.7 \%^{*}$ & $4 \pm 0.7 \%^{*}$ \\
\hline \multirow[t]{2}{*}{14} & $1289 \pm 11$ & $1055 \pm 20$ & $1079 \pm 14$ & $1182 \pm 13^{*}$ & $1192 \pm 7^{*}$ \\
\hline & - & $18 \pm 1.3 \%$ & $16 \pm 1.4 \%$ & $8 \pm 0.7 \%^{*}$ & $7 \pm 1.0 \% *$ \\
\hline \multirow[t]{2}{*}{28} & $1283 \pm 8$ & $952 \pm 21$ & $983 \pm 17$ & $1021 \pm 15^{*}$ & $1091 \pm 27^{*}$ \\
\hline & - & $26 \pm 1.2 \%$ & $23 \pm 1.4 \%$ & $20 \pm 1.3 \%^{*}$ & $15 \pm 2.3 \%^{*}$ \\
\hline
\end{tabular}

*, $p<0.05$ when compared with the saline-treated group

neurons having different functions, namely, somatic efferents of the HN and visceral efferents of the DMN $[23,27]$. In agreement with these findings, the present results demonstrated a peak of PNCI-induced NADPH$\mathrm{d} / \mathrm{nNOS}$ expression in motor neurons of the $\mathrm{HN}$ at 7 days, whereas those of the DMN persisted up to 28 days (Figures 2, 3 and 4). This difference between the motor neurons of the HN and DMN remains to be resolved but may be due to differences in their regenerative capacity. Previous tracing study with horseradish peroxidase demonstrated that the majority of axotomized hypoglossal motor neurons re-innervated tongue muscles at 14 days post-PNI [16]. During re-innervation, neurotrophic factors may stimulate motor neuron regeneration and thereby ensure the decrease in NADPH-d/nNOS expression in successful regenerating motor neurons $[17,58,59]$. Thus, to the extent that NADPH-d/nNOS expression is correlated with the degree of functional recovery by injured motor neurons, down-regulation of NADPH-d/ nNOS expression indicates a predictable functional recovery of motor neurons in the crushed HN. Furthermore, the suppressive NADPH-d/nNOS expression seen in rats treated with high-doses EGCG (25 and $50 \mathrm{mg} / \mathrm{kg}$ ) at all time points post-PNI implied that high-dose EGCG facilitated functional recovery of the damaged HN. In contrast, the current investigation also found that the sustained expression of NADPH-d/nNOS in damaged motor neurons of the DMN adversely affected their functional recovery [17]. Our present evidences may echo the finding that axonal regeneration appeared to be absent in visceral motor neurons of the DMN [17]. However, in view of the fact that the viability of surviving motor neurons following PNI depended on their proximity to injured axons [51,60], the injury sites of the hypoglossal and vagus nerves should be seriously considered. In our present study, the nerve crush at the cervical level of the vagus nerve relative to that of the hypoglossal nerve may have been proximal enough to cause severe damage to DMN motor neurons.

\section{Conclusion}

The current study demonstrated morphologically and quantitatively that high-doses ( 25 and $50 \mathrm{mg} / \mathrm{kg}$ ) EGCG can reduce PNCI-induced NADPH-d/nNOS expression in the $\mathrm{HN}$ and DMN motor neurons. This novel finding not only improved our understanding of the functional role of NO in neuronal damage, but also provided an EGCG neuroprotective strategy to suppress nNOS-mediated toxicity in the $\mathrm{HN}$ and DMN motor neurons damaged by PNCI.

\section{Acknowledgements}

We are grateful to Professor C. H. Wu for his critical reviews and comments. This study was supported in part by research grants (NSC 99-2320-B039-019MY3 and CMU96-133) to I. H. Wei from the National Science Council and 
China Medical University, Taiwan and (NSC 91-2320-B002-128 and NSC 922320-B002-100) to Professor J. Y. Shieh from the National Science Council, Taiwan.

\section{Author details}

${ }^{1}$ Department of Anatomy and Cell Biology, College of Medicine, China Medical University, Taichung, Taiwan. ${ }^{2}$ Department of Psychiatry, China Medical University and Hospital, Taichung, Taiwan. ${ }^{3}$ Department of Neurology, Taichung Tzu Chi General Hospital, Taichung, Taiwan. ${ }^{4}$ Department of Anatomy and Cell Biology, College of Medicine, National Taiwan University, Taipei, Taiwan.

\section{Authors' contributions}

$\mathrm{IHW}, \mathrm{CCH}, \mathrm{MHT}$ and JYS conceived this experiment. Animal studies were performed by IHW, HCT and CYT. Immunohistochemistry was performed by IHW, HCT, MHT and CYT. Data acquisition, analysis and manuscript preparation were performed by $I H W, H C T, C C H, M H T$ and JYS. The manuscript was finally edited by IHW, CCH, MHT and JYS. All authors had read and approved the final submitted and published versions.

Received: 3 November 2010 Accepted: 1 June 2011

Published: 1 June 2011

\section{References}

1. Lieberman AR: The axon reaction: a review of the principal features of perikaryal responses to axon injury. Int Rev Neurobiol 1971, 14:49-124.

2. Aldskogius $H$, Barron $K D$, Regal $R$ : Axon reaction in dorsal motor vagal and hypoglossal neurons of the adult rat. Light microscopy and RNAcytochemistry. J Comp Neurol 1980, 193:165-177.

3. Grafstein B: The nerve cell body response to axotomy. Exp Neurol 1975, 48:32-51.

4. Halliwell B: Reactive oxygen species and the central nervous system. J Neurochem 1992, 59:1609-1623.

5. Palmer RM, Ferrige AG, Moncada S: Nitric oxide release accounts for the biological activity of endothelium-derived relaxing factor. Nature 1987, 327:524-526

6. Moncada S, Palmer RM, Gryglewski RJ: Mechanism of action of some inhibitors of endothelium-derived relaxing factor. Proc Natl Acad Sci USA 1986, 83:9164-9168.

7. Marletta MA: Nitric oxide synthase: aspects concerning structure and catalysis. Cell 1994, 78:927-930.

8. Forstermann U, Schmidt HH, Pollock JS, Sheng H, Mitchell JA, Warner TD, et al: Isoforms of nitric oxide synthase. Characterization and purification from different cell types. Biochem Pharmacol 1991, 42:1849-1857.

9. Szabo C: Physiological and pathophysiological roles of nitric oxide in the central nervous system. Brain Res Bull 1996, 41:131-141.

10. Beckman JS, Beckman TW, Chen J, Marshall PA, Freeman BA: Apparent hydroxyl radical production by peroxynitrite: implications for endothelial injury from nitric oxide and superoxide. Proc Natl Acad Sci USA 1990, 87:1620-1624.

11. Beckman JS, Koppenol WH: Nitric oxide, superoxide, and peroxynitrite: the good, the bad, and ugly. Am J Physiol 1996, 271:C1424-C1437.

12. Pryor WA, Squadrito GL: The chemistry of peroxynitrite: a product from the reaction of nitric oxide with superoxide. Am J Physiol 1995, 268: L699-L722.

13. Jia YS, Wang XA, Ju G: Nitric oxide synthase expression in vagal complex following vagotomy in the rat. Neuroreport 1994, 5:793-796.

14. Ruan RS, Leong SK, Yeoh KH: The role of nitric oxide in facial motoneuronal death. Brain Res 1995, 698:163-168.

15. Zochodne DW, Misra M, Cheng C, Sun H: Inhibition of nitric oxide synthase enhances peripheral nerve regeneration in mice. Neurosci Lett 1997, 228:71-74.

16. Yu WH, Srinivasan R: Effect of testosterone and 5 alphadihydrotestosterone on regeneration of the hypoglossal nerve in rats. Exp Neurol 1981, 71:431-435.

17. Yu WH: Regulation of nitric oxide synthase expression in motoneurons following nerve injury. Dev Neurosci 1997, 19:247-254.

18. Wu W: Expression of nitric-oxide synthase (NOS) in injured CNS neurons as shown by NADPH diaphorase histochemistry. Exp Neurol 1993, 120:153-159.
19. Higuchi $Y$, Hattori $H$, Hattori R, Furusho K: Increased neurons containing neuronal nitric oxide synthase in the brain of a hypoxic-ischemic neonatal rat model. Brain Dev 1996, 18:369-375.

20. Chang HM, Ling EA, Lue JH, Wen CY, Shieh JY: Melatonin attenuates neuronal NADPH-d/NOS expression in the hypoglossal nucleus of adult rats following peripheral nerve injury. Brain Res 2000, 873:243-251.

21. Chang HM, Ling EA, Chen CF, Lue H, Wen CY, Shieh JY: Melatonin attenuates the neuronal NADPH-d/NOS expression in the nodose ganglion of acute hypoxic rats. J Pineal Res 2002, 32:65-73.

22. Chang HM, Huang YL, Lan CT, Wu UI, Hu ME, Youn SC: Melatonin preserves superoxide dismutase activity in hypoglossal motoneurons of adult rats following peripheral nerve injury. J Pineal Res 2008, 44:172-180.

23. Chang HM, Lue JH, Wen CY, Shieh JY: Axotomy along with hypoxia enhances the neuronal NADPH-d/NOS expression in lower brain stem motor neurons of adult rats. Exp Neurol 2001, 171:116-126.

24. Chang HM, Liao WC, Lue JH, Wen CY, Shieh JY: Upregulation of NMDA receptor and neuronal NADPH-d/NOS expression in the nodose ganglion of acute hypoxic rats. J Chem Neuroanat 2003, 25:137-147.

25. Guo Y, Ward ME, Beasjours S, Mori M, Hussain SN: Regulation of cerebellar nitric oxide production in response to prolonged in vivo hypoxia. $J$ Neurosci Res 1997, 49:89-97.

26. Wei $I H$, Wu YC, Wen CY, Shieh JY: Green tea polyphenol (-)-epigallocatechin gallate attenuates the neuronal NADPH-d/nNOS expression in the nodose ganglion of acute hypoxic rats. Brain Res 2004, 999:73-80.

27. Wei $\mathrm{H}$, Huang CC, Tseng $\mathrm{CY}$, Chang HM, Tu HC, Tsai MH, et al: Mild hypoxic preconditioning attenuates injury-induced NADPH-d/nNOS expression in brainstem motor neurons of adult rats. J Chem Neuroanat 2008, 35:123-132

28. Wei IH, Huang CC, Chang HM, Tseng CY, Tu HC, Wen CY, et al: Neuronal $\mathrm{NADPH}-\mathrm{d} / \mathrm{NOS}$ expression in the nodose ganglion of severe hypoxic rats with or without mild hypoxic preconditioning. J Chem Neuroanat 2005, 29:149-156.

29. Reiter RJ: Oxidative damage in the central nervous system: protection by melatonin. Prog Neurobiol 1998, 56:359-384.

30. Hanasaki Y, Ogawa S, Fukui S: The correlation between active oxygens scavenging and antioxidative effects of flavonoids. Free Radic Biol Med 1994, 16:845-850.

31. Haenen GR, Paquay JB, Korthouwer RE, Bast A: Peroxynitrite scavenging by flavonoids. Biochem Biophys Res Commun 1997, 236:591-593.

32. Ho CT, Chen Q, Shi H, Zhang KQ, Rosen RT: Antioxidative effect of polyphenol extract prepared from various Chinese teas. Prev Med 1992, 21:520-525.

33. Zhao BL, Li XJ, He RG, Cheng SJ, Xin WJ: Scavenging effect of extracts of green tea and natural antioxidants on active oxygen radicals. Cell Biophys 1989, 14:175-185.

34. van Acker SA, Tromp MN, Haenen GR, van d V, Bast A: Flavonoids as scavengers of nitric oxide radical. Biochem Biophys Res Commun 1995, 214:755-759.

35. Kelly MR, Geigerman CM, Loo G: Epigallocatechin gallate protects U937 cells against nitric oxide-induced cell cycle arrest and apoptosis. J Cell Biochem 2001, 81:647-658.

36. Kondo K, Kurihara M, Miyata N, Suzuki T, Toyoda M: Scavenging mechanisms of (-)-epigallocatechin gallate and (-)-epicatechin gallate on peroxyl radicals and formation of superoxide during the inhibitory action. Free Radic Biol Med 1999, 27:855-863.

37. Guo Q, Zhao B, Li M, Shen S, Xin W: Studies on protective mechanisms of four components of green tea polyphenols against lipid peroxidation in synaptosomes. Biochim Biophys Acta 1996, 1304:210-222.

38. Lin YL, Lin JK: (-)-Epigallocatechin-3-gallate blocks the induction of nitric oxide synthase by down-regulating lipopolysaccharide-induced activity of transcription factor nuclear factor-kappaB. Mol Pharmacol 1997 52:465-472.

39. Chan MM, Fong D, Ho CT, Huang HI: Inhibition of inducible nitric oxide synthase gene expression and enzyme activity by epigallocatechin gallate, a natural product from green tea. Biochem Pharmacol 1997, 54:1281-1286.

40. Sano M, Takahashi $Y$, Yoshino K, Shimoi K, Nakamura Y, Tomita I, et al: Effect of tea (Camellia sinensis L.) on lipid peroxidation in rat liver and kidney: a comparison of green and black tea feeding. Biol Pharm Bull $1995,18: 1006-1008$ 
41. Suganuma M, Okabe S, Oniyama M, Tada Y, Ito H, Fujiki H: Wide distribution of $[3 \mathrm{H}](-)$-epigallocatechin gallate, a cancer preventive tea polyphenol, in mouse tissue. Carcinogenesis 1998, 19:1771-1776.

42. Lin LC, Wang MN, Tseng TY, Sung JS, Tsai TH: Pharmacokinetics of (-)-epigallocatechin-3-gallate in conscious and freely moving rats and its brain regional distribution. J Agric Food Chem 2007, 55:1517-1524.

43. Vincent $\mathrm{SR}$, Kimura $\mathrm{H}$ : Histochemical mapping of nitric oxide synthase in the rat brain. Neuroscience 1992, 46:755-784.

44. Scherer-Singler U, Vincent SR, Kimura H, McGeer EG: Demonstration of a unique population of neurons with NADPH-diaphorase histochemistry. J Neurosci Methods 1983, 9:229-234

45. Vige X, Carreau A, Scatton B, Nowicki JP: Antagonism by NG-nitro-Larginine of L-glutamate-induced neurotoxicity in cultured neonatal rat cortical neurons. Prolonged application enhances neuroprotective efficacy. Neuroscience 1993, 55:893-901.

46. Chen YS, Tseng FY, Tan CT, Lin-Shiau SY, Hsu CJ: Effects of methylprednisolone on nitric oxide formation and survival of facial motor neurons after axotomy. Brain Res 2008, 1197:23-31.

47. Moncada S, Palmer RM, Higgs EA: Nitric oxide: physiology, pathophysiology, and pharmacology. Pharmacol Rev 1991, 43:109-142

48. Snyder SH, Bredt DS: Biological roles of nitric oxide. Sci Am 1992, 266:68-7.

49. Kristensson K, Aldskogius M, Peng ZC, Olsson T, Aldskogius H, Bentivoglio M: Co-induction of neuronal interferon-gamma and nitric oxide synthase in rat motor neurons after axotomy: a role in nerve repair or death? J Neurocytol 1994, 23:453-459.

50. Wu W: Potential roles of gene expression change in adult rat spinal motoneurons following axonal injury: a comparison among c-jun, offaffinity nerve growth factor receptor (LNGFR), and nitric oxide synthase (NOS). Exp Neurol 1996, 141:190-200.

51. Wu W, Li L: Inhibition of nitric oxide synthase reduces motoneuron death due to spinal root avulsion. Neurosci Lett 1993, 153:121-124.

52. Simon DK, Standaert DG: Neuroprotective therapies. Med Clin North Am 1999, 83:509-23, viii.

53. Jung JY, Han CR, Jeong YJ, Kim HJ, Lim HS, Lee KH, et al: Epigallocatechin gallate inhibits nitric oxide-induced apoptosis in rat PC12 cells. Neurosci Lett 2007, 411:222-227.

54. Lee $H$, Bae JH, Lee SR: Protective effect of green tea polyphenol EGCG against neuronal damage and brain edema after unilateral cerebral ischemia in gerbils. J Neurosci Res 2004, 77:892-900.

55. Lee S, Suh S, Kim S: Protective effects of the green tea polyphenol (-)-epigallocatechin gallate against hippocampal neuronal damage after transient global ischemia in gerbils. Neurosci Lett 2000, 287:191-194.

56. Endoh M, Maiese $\mathrm{K}$, Wagner J: Expression of the inducible form of nitric oxide synthase by reactive astrocytes after transient global ischemia. Brain Res 1994, 651:92-100.

57. O'Neill MJ, Hicks C, Ward M, Panetta JA: Neuroprotective effects of the antioxidant LY231617 and NO synthase inhibitors in global cerebral ischaemia. Brain Res 1997, 760:170-178.

58. Lowrie MB, Vrbova G: Dependence of postnatal motoneurones on their targets: review and hypothesis. Trends Neurosci 1992, 15:80-84.

59. Wu W, Li L, Yick LW, Chai H, Xie Y, Yang Y, et al: GDNF and BDNF alter the expression of neuronal NOS, c-Jun, and p75 and prevent motoneuron death following spinal root avulsion in adult rats. J Neurotrauma 2003, 20:603-612.

60. Gu Y, Spasic $Z, W u$ W: The effects of remaining axons on motoneuron survival and NOS expression following axotomy in the adult rat. Dev Neurosci 1997, 19:255-259.

doi:10.1186/1471-2202-12-52

Cite this article as: Wei et al:: (-)-Epigallocatechin gallate attenuates $\mathrm{NADPH}-\mathrm{d} / \mathrm{nNOS}$ expression in motor neurons of rats following peripheral nerve injury. BMC Neuroscience 2011 12:52.

\section{Submit your next manuscript to BioMed Central and take full advantage of:}

- Convenient online submission

- Thorough peer review

- No space constraints or color figure charges

- Immediate publication on acceptance

- Inclusion in PubMed, CAS, Scopus and Google Scholar

- Research which is freely available for redistribution

Submit your manuscript at www.biomedcentral.com/submit 